\title{
Short communication: A study of Lactobacillus isolates' adherence to and influence on membrane integrity of human Caco-2 cells
}

\author{
Neethu M. Jose, ${ }^{*}$ Craig R. Bunt, $\dagger^{1}$ Arlene McDowell, $\ddagger$ Jasper Z. S. Chiu, $\ddagger$ and Malik A. Hussain* ${ }^{* 1}$ \\ *Department of Wine, Food and Molecular Biosciences, and \\ †Department of Agriculture, Lincoln University, Lincoln 7647, New Zealand \\ ¥School of Pharmacy, University of Otago, PO Box 56, Dunedin 9054, New Zealand
}

\section{ABSTRACT}

The selection criteria of ideal probiotic bacteria are complex and involve many factors. One key criterion is based on the ability of the probiotic bacteria to adhere to the epithelial lining of the gastrointestinal tract. The objective of this study was to evaluate and compare the adherence and influence on membrane integrity of 2 selected lactobacilli isolates - Lactobacillus rhamnosus MI13 (dairy food origin) and L. plantarum RC2 (bovine rumen origin) - to Caco-2 cells in the presence and absence of Escherichia coli. The adhesion and influence on membrane integrity properties of the 2 Lactobacillus isolates were compared with Escherichia coli, a human commensal bacterium. From the adhesion studies, we concluded that the bovine rumen isolate exhibited better adherence to Caco-2 cells than the dairy food isolate. In contrast, the dairy food isolate better protected the Caco-2 monolayer from damage induced by ethanol.

Key words: lactobacilli, Escherichia coli, Caco-2 cells, adherence, permeability

\section{Short Communication}

An effective probiotic bacterium must survive and function under a variety of different environmental and physiological conditions. The ideal probiotic must possess high viability and maintain viability throughout processing conditions, be resistant to acidic conditions, be tolerant of bile salts, be able to interact and trigger immune cells in the gut, and be nonpathogenic and genetically stable (Gupta and Garg, 2009). Key criteria for selecting a probiotic bacterium include adherence to the intestinal epithelial lining and the ability to colonize the gut (Alander et al., 1999; Mattila-Sandholm et al., 2009). After ingestion of probiotic bacteria, the

\footnotetext{
Received March 22, 2017.

Accepted July 6, 2017.

${ }^{1}$ Corresponding authors: craig.bunt@lincoln.ac.nz and malikaltaf_ ft@hotmail.com
}

survival and persistence of the probiotic bacteria in the gut relies on host-bacteria interactions. Successful adhesion of probiotic bacteria to the gut wall is believed to help establish colonization in the gut (Gueimonde and Salminen, 2006).

Understanding the mechanisms responsible for probiotic bacterial adhesion in vivo is challenging. Caco-2 cells (a human adenocarcinoma cell line) resemble the enterocytes lining the intestine and represent a widely used model for assessment of interactions in the human gut in preclinical studies. This has led to their use in the study of bacterial adherence in vitro (Kleeman and Klaenhammer, 1982; Conway et al., 1987; Duary et al., 2011). The Caco- 2 cell line is an excellent model to understand how probiotic bacteria interact with the intestinal epithelial lining (Wang et al., 2008; Moussavi and Adams, 2010). Caco-2 cells can also be used to investigate how probiotic bacteria compete with other human commensals for attachment sites in the gut (Liu et al., 2003; Thirabunyanon et al., 2009).

Integrity of the intestinal barrier plays a significant role in maintenance of good health. This is attributed to the formation of tight junctions between adjacent intestinal epithelial cells, and restricting free passage of nutrients and pathogens into the systemic circulationselective absorption of particulates and solutions from the lumen of the gastrointestinal tract (Schneeberger and Lynch, 2004). A compromise in the intestinal barrier makes it "leaky," and would likely result in infections when bacteria are able to traverse the barrier of the gut epithelium (Anderson et al., 2010). A change in the integrity of the Caco-2 cell monolayer, which reflects the intestinal barrier function of the epithelium cells, can be determined and measured in vitro in terms of trans-epithelial electrical resistance (TEER) of the Caco-2 cell monolayer (Klingberg et al., 2005).

We previously assessed 10 lactobacilli isolates, 5 each from dairy food products and bovine rumen contents, for their hydrophobicity (Jose et al., 2015) using the bacterial adherence to hydrocarbons (BATH) method (Bunt et al., 1993). From our earlier study, it was 
evident that the bovine rumen isolates exhibited better adhesion to dichloromethane compared with dairy food isolates (Jose et al., 2015). In the present study, 2 of the 10 isolates, 1 from dairy food and 1 from bovine rumen, were further investigated for their adhesion to and effect on the integrity of a Caco-2 cell monolayer in vitro. Each isolate was selected from its group based on sugar fermentation capability, resistance to bile salts, resistance to low $\mathrm{pH}$, adhesion, antibiotic resistance, and ability to inhibit pathogens.

Lactobacillus rhamnosus MI13 was isolated from a dairy food product and Lactobacillus plantarum $\mathrm{RC} 2$ was isolated from the bovine rumen by Jose et al. (2015) as part of a wider program to isolate and characterize potential probiotic bacteria. Inhibition of pathogens, resistance to antibiotics, biosafety (absence of hemolytic activity), adhesion (BATH test), carbohydrate fermentation, survival at low $\mathrm{pH}$, and high bile salt concentration characteristics of L. rhamnosus MI13 and L. plantarum $\mathrm{RC} 2$ suggested that further evaluation of these isolates would be warranted. A comparison of DNA sequence using the BLASTN nucleotide database (https://blast.ncbi.nlm.nih.gov/Blast.cgi) gave the best match of MI13 with L. rhamnosus strain LOCK908 and RC2 with L. plantarum strain 16 (Jose et al., 2015). The 2 lactobacilli isolates were cultured in de Man, Rogosa, Sharpe (MRS) broth (Oxoid, Basingstoke, $\mathrm{UK})$ at $\mathrm{pH} 6.4 \pm 0.2$, and a nonpathogenic Escherichia coli isolate (The Institute of Environmental Science and Research, Porirua, New Zealand) was cultured in brain heart infusion (BHI) broth (Oxoid) at $\mathrm{pH} 7.4 \pm 0.2$. The cultures were incubated at $37^{\circ} \mathrm{C}$ overnight. After incubation, the cultures were centrifuged at 16,000 $\times$ $g$ for $10 \mathrm{~min}$. The cells were washed and suspended in PBS at $\mathrm{pH} 7.4$ and adjusted to an optical density of 2.0 (at $600 \mathrm{~nm}$ ).

The Caco-2 cell line (passage 71 to 84 ) used for the adhesion studies was propagated and maintained at the University of Otago (Dunedin, New Zealand). Caco-2 cells were cultured in BD Falcon cell culture flasks with a surface area of $75 \mathrm{~cm}^{2}$ (BD Biosciences, Bedford, MA) following standard protocols (Gao et al., 2000). The cells were maintained at $37^{\circ} \mathrm{C}$ in $10 \% \mathrm{CO}_{2}$ and $90 \%$ air. Cells were fed on alternate days with 15 $\mathrm{mL}$ of Dulbecco's modified Eagle medium (DMEM) supplemented with $10 \%$ fetal bovine serum (Moregate Biotech, Bulimba, QLD, Australia), 1\% NEAA (100×), and $1 \%$ penicillin-streptomycin $(10,000 \mathrm{U} / \mathrm{mL})$. At $70 \%$ confluency, cells were detached from the flask with 2 $\mathrm{mL}$ of trypLE Express Enzyme $(1 \times$; Thermo Fisher, Auckland, New Zealand) for seeding. All cell culture media were sourced from Gibco Life Technologies Corp. (New York, NY).
Caco-2 cells were seeded on Transwell permeable supports (Corning Inc., Corning, NY) at 50,000 cells/ well $(0.5 \mathrm{~mL}$ of 100,000 cells $/ \mathrm{mL})$ for 21 to $28 \mathrm{~d}$ (to obtain a fully differentiated monolayer) for monolayer integrity studies. The cells, grown on the permeable support, were fed on alternate days with fresh supplemented DMEM medium as previously described (0.5 $\mathrm{mL}$ in the donor compartment and $1.5 \mathrm{~mL}$ in the receiver compartment).

Three-week-old Caco-2 cells were seeded with supplemented DMEM in Transwell membrane tissue culture plates. The 2 lactobacilli isolates, L. rhamnosus MI13 and L. plantarum $\mathrm{RC} 2$, and $E$. coli were added individually and each lactobacilli isolate was added in combination with $E$. coli into the wells of the tissue culture plates. Plates were then incubated at $37^{\circ} \mathrm{C}$ in $10 \% \mathrm{CO}_{2}$ and $90 \%$ air. After incubation, the Caco- 2 monolayers were washed with sterile PBS several times to remove unattached bacteria. Samples were fixed with methanol and placed on a glass microscope slide followed by Gram staining. The Gram-stained slides were observed using an oil-immersion objective $(100 \times)$ and a light microscope to count the average number of bacteria that had adhered to the Caco- 2 cells. The bacteria in 25 microscopic fields were counted for each sample (Liu et al., 2003; Thirabunyanon et al., 2009).

The matured Caco-2 cell monolayers (cultured for 21 to 28 d) grown on Transwell inserts were washed with diluted DMEM (1:1 ratio of complete DMEM to PBS) twice. Then, the cells were incubated with $0.5 \mathrm{~mL}$ and $1.5 \mathrm{~mL}$ of diluted DMEM in the donor and receiver compartments, respectively, of Transwell inserts and maintained at $37^{\circ} \mathrm{C}$ for $30 \mathrm{~min}$ before the membrane integrity study. The diluted DMEM in the donor compartment was then replaced with fresh diluted DMEM (control) or diluted DMEM containing 1\% ethanol alone (positive control) or E. coli $(0.4 \mathrm{~mL})$, L. rhamnosus MI13 $(0.4 \mathrm{~mL})$, L. plantarum RC2 $(0.4 \mathrm{~mL})$, E. coli $(0.4$ $\mathrm{mL})$ and L. rhamnosus MI13 $(0.4 \mathrm{~mL})$, or E. coli $(0.4$ $\mathrm{mL}$ ) and L. plantarum $\mathrm{RC} 2(0.4 \mathrm{~mL})$. Co-incubation with the Caco-2 cell monolayers was carried out at $37^{\circ} \mathrm{C}$ for $90 \mathrm{~min}$. Then, TEER measurements were taken at 2, 30, 60, and 90 min using a Millicell-ERS volt-ohm meter (EMD Millipore Corp., Billerica, MA).

Statistical analysis was performed using a general linear model ANOVA (Minitab version 17, Minitab Inc., State College, PA). Tukey's test was used to undertake pairwise comparisons for significance at $P<0.05$ for the isolates in the adhesion and permeability studies.

Using light microscopy in combination with Gram staining, we were able to distinguish small lactobacilli isolates (violet) and E. coli (pink) from larger pink Caco-2 cells (Figure 1). When each bacterium was 
added individually to Caco-2 cells, E. coli showed the maximum average adherence of 24 bacteria per Caco- 2 cell (Figure 2). The bovine rumen isolate (L. plantarum $\mathrm{RC} 2$ ) adhered less to Caco-2 cells, with on average 6 bacteria per Caco-2 cell. The dairy food isolate $(L$. rhamnosus MI13) showed the lowest average adherence when bacteria were applied individually to Caco-2 cells, with only 3 bacteria per Caco-2 cell. However, when lactobacilli isolates were added to Caco-2 cells in combination with $E$. coli, the average number of $E$. coli that adhered to Caco-2 cells was reduced compared with $E$. coli alone. The average number of $E$. coli adhered to Caco- 2 cells in the presence of the bovine rumen isolate (L. plantarum $\mathrm{RC} 2$ ) or dairy food isolate (L. rhamnosus MI13) was significantly reduced $(P<0.05)$. In the presence of $E$. coli, adherence of the dairy food isolate (L. rhamnosus MI13) to Caco-2 cells was significantly $(P<0.05)$ reduced. However, the presence of $E$. coli did not have any effect $(P>0.05)$ on the adherence of the bovine rumen isolate (L. rhamnosus $\mathrm{RC} 2$ ) to Caco2 cells.

Co-incubation with $1 \%$ ethanol alone (positive control) was observed to rapidly and significantly $(P<$
0.05) reduce the TEER value compared with the (negative) control without treatment (Figure 3). By $30 \mathrm{~min}$, the TEER of $1 \%$ ethanol-treated Caco-2 monolayers had reduced to approximately $450 \Omega / \mathrm{cm}^{2}$ and remained at this level for the duration of the experiment. The TEER value was significantly $(P<0.05)$ greater when Caco-2 membranes were co-incubated with L. plantarum $\mathrm{RC} 2$, L. rhamnosus MI13, or E. coli compared with Caco-2 cell monolayers exposed to $1 \%$ ethanol in the first 30 min after treatment. By $60 \mathrm{~min}$, the TEER of Caco-2 monolayer was significantly $(P<0.05)$ greater when co-incubated with $L$. plantarum RC2, E. coli, or E. coli and L. plantarum RC2 compared with the $1 \%$ ethanol alone treatments. At $90 \mathrm{~min}$ after treatment, the TEER of L. plantarum RC2 and E. coli co-incubated Caco-2 monolayer were significantly greater than all other treatments.

Gram-positive and gram-negative bacteria are both present in the human gut microbiota. Lactobacilli are gram-positive, health-promoting bacteria that exist in relatively large numbers in the gut. Evaluating their adherence to the gut wall might provide insight into how they function in vivo to promote health benefits

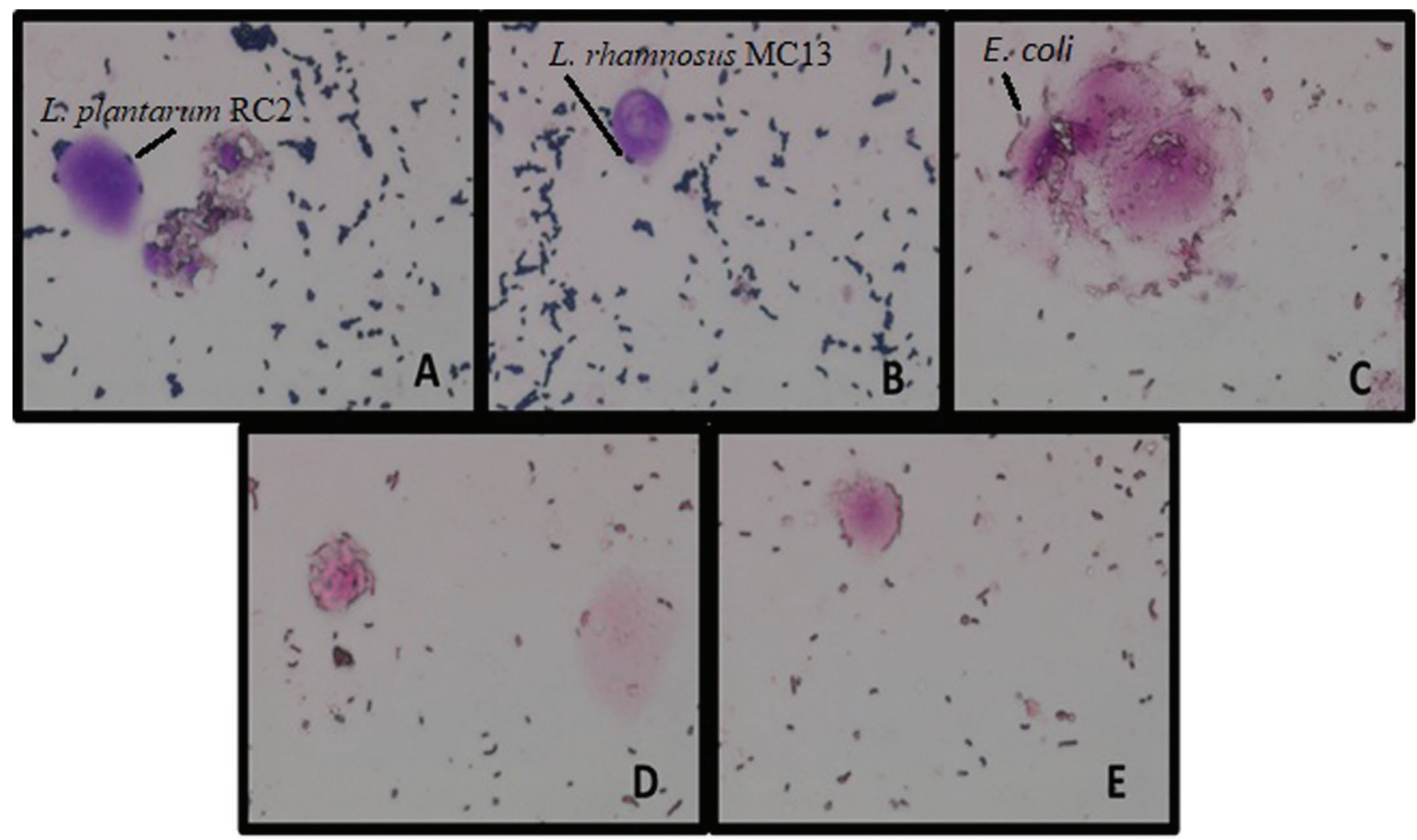

Figure 1. Gram stain images showing adhesion of lactobacilli isolates and Escherichia coli on 3-wk-old Caco-2 cells observed under oil immersion $(100 \times)$. (A) Bovine rumen isolate Lactobacillus plantarum RC2; (B) dairy food isolate Lactobacillus rhamnosus MI13; (C) E. coli; (D) bovine rumen isolate L. plantarum $\mathrm{RC} 2$ and E. coli; (E) dairy food isolate L. rhamnosus MI13 and E. coli. Color version available online. 


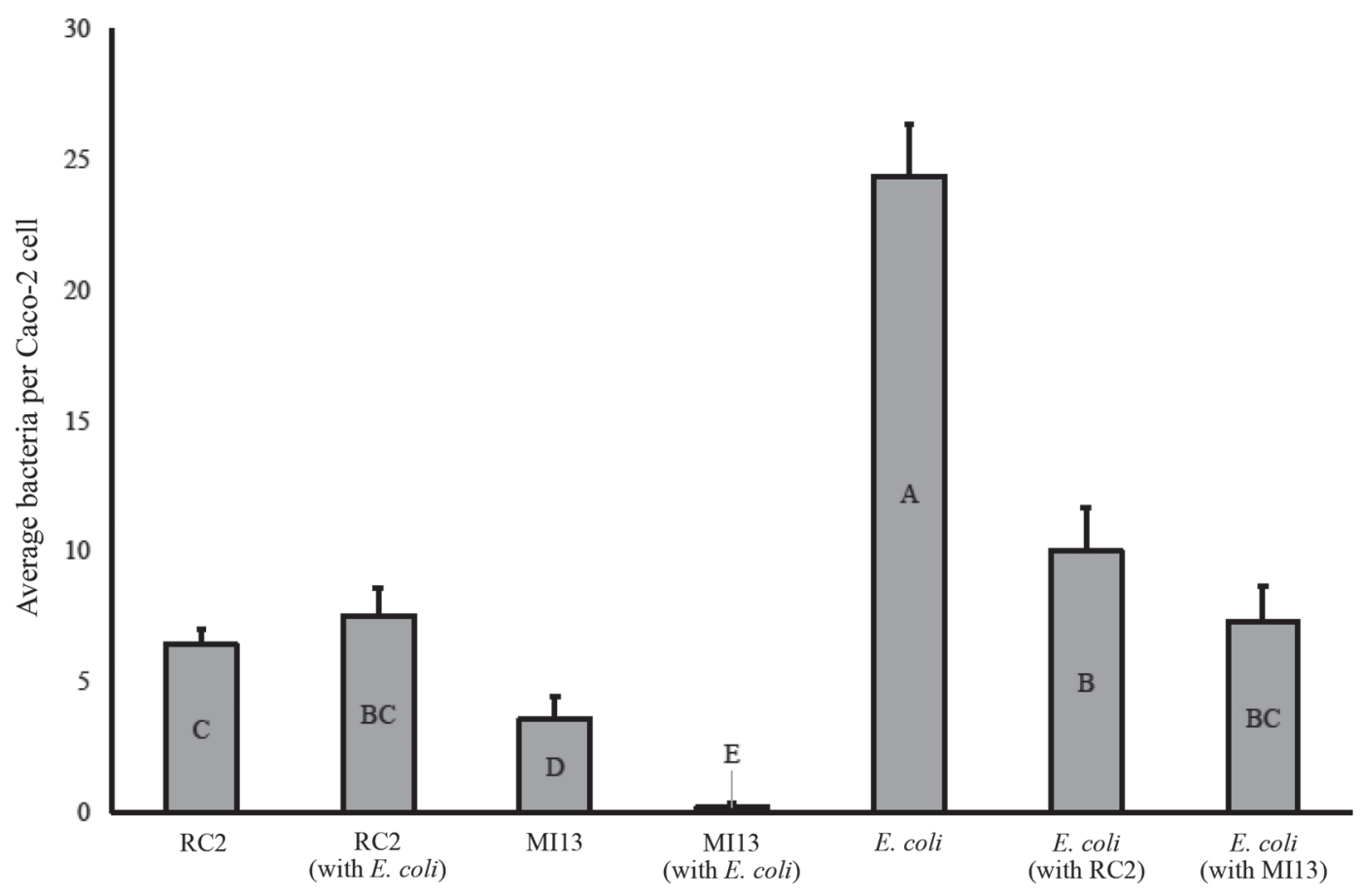

Figure 2. Average adhesion of lactobacilli isolates and Escherichia coli to 3-wk-old Caco-2 cells. Error bars are standard error of the mean $(\mathrm{n}=4)$; columns that do not share a letter $(\mathrm{A}-\mathrm{E})$ are significantly different $(P<0.05)$.

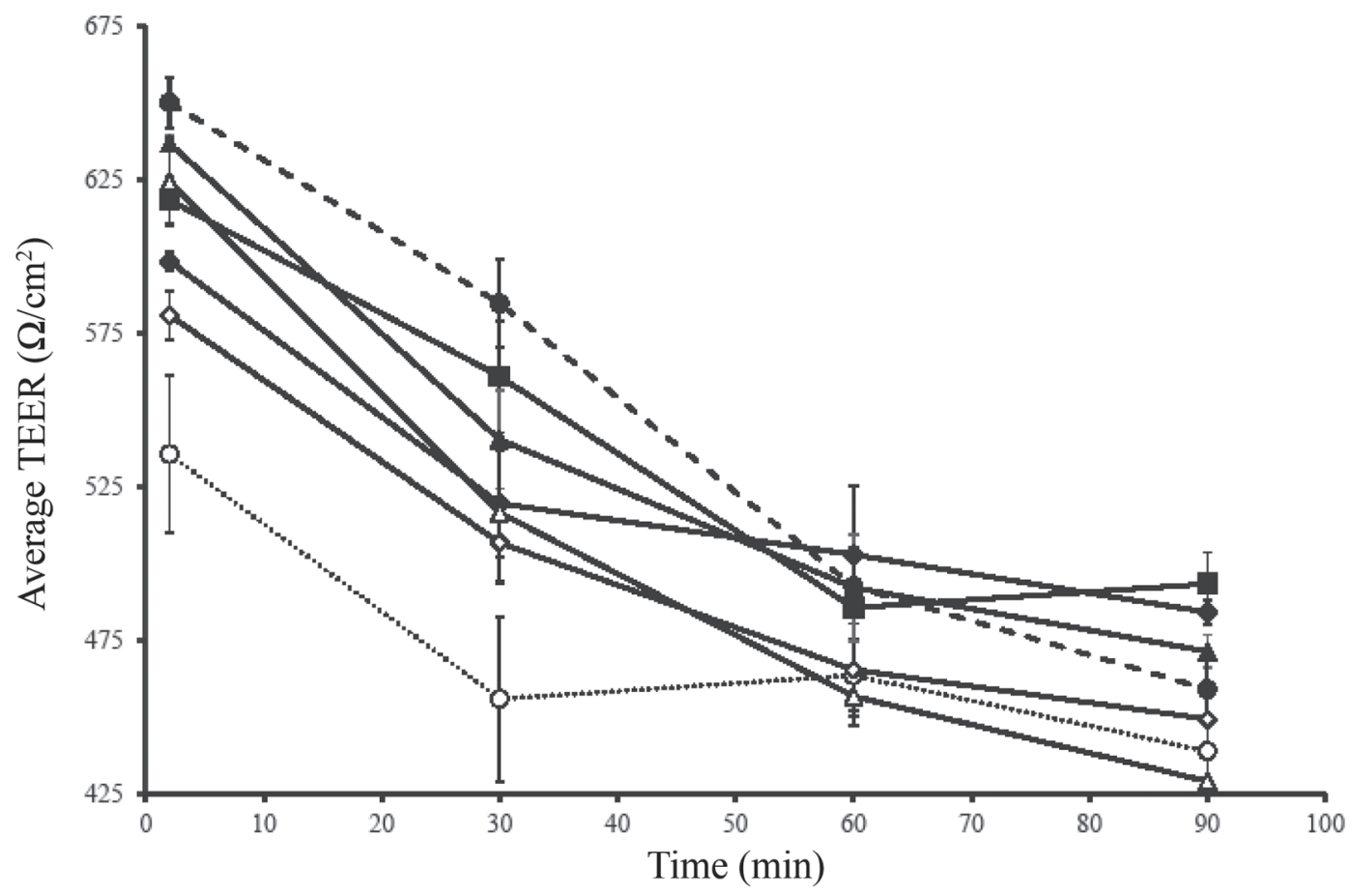

Figure 3. Average trans-epithelial electrical resistance (TEER; $\Omega / \mathrm{cm}^{2}$ ) measurements of Caco-2 monolayer $2,30,60$, and 90 min after treatment with Lactobacillus rhamnosus MI13 or Lactobacillus plantarum RC2, with or without Escherichia coli after treatment with $1 \%$ ethanol, and a control without treatment at $37^{\circ} \mathrm{C}$ : negative control ( $\bullet$, dashed line); ethanol $1 \%$ ( $\bigcirc$, dotted line); ethanol $1 \%$ and $E$. coli $(\mathbf{\square})$, ethanol $1 \%$ and L. rhamnosus MI13 ( ), ethanol $1 \%$ and L. plantarum RC2 $(\diamond)$, ethanol 1\%, E. coli, and L. rhamnosus MI13 (ム), ethanol $1 \%$, E. coli, and L. plantarum $\mathrm{RC} 2(\Delta)$. Error bars are standard error of the mean $(\mathrm{n}=3)$. 
in the host. Caco-2 cells are an ideal model to study this phenomenon in vitro. In this study, the 2 lactobacilli isolates were capable of adhering to Caco-2 cells, albeit at varying degrees. The bovine rumen isolate, $L$. plantarum $\mathrm{RC} 2$, showed the highest adherence, and the dairy food isolate, L. rhamnosus MI13, showed the least adherence. Incubating the lactobacilli isolates on Caco2 cells in the presence of $E$. coli helped us to understand how the lactobacilli isolates compete for binding sites with a human commensal. Based on our results, it was evident that the rumen isolate $L$. plantarum $\mathrm{RC} 2$ was unaffected by the presence of $E$. coli. However, for the dairy food isolate, L. rhamnosus MI13, adherence was greatly lowered in the presence of $E$. coli. It was previously reported that $L$. plantarum of fecal origin had a higher adherence rate to Caco-2 cells, and a food isolate, Latobacillus delbrueckii ssp. bulgaricus showed a lower adherence rate (Duary et al., 2011). Further, it has been shown that within strains of $L$. delbrueckii ssp. bulgaricus, there is a difference in adherence to Caco-2 cells, which is attributed to the production of exopolysaccharide (EPS). The strain that produced more EPS had a higher survival rate and showed better adherence under gastrointestinal tract conditions compared with the low-EPS-producing B2 strain (Darilmaz et al., 2011). This suggests that the adherence property of Lactobacillus species is strain specific (Chauvière et al., 1992) and origin specific. Perhaps the ability of the bovine rumen isolates to adhere better to Caco- 2 cells alone or in the presence of $E$. coli is indicative of adaptations that reflect the stressful environment of the bovine rumen.

From the membrane integrity study, we concluded that the presence of the bovine rumen isolate did not affect the permeability of the intestinal lining. On the other hand, the dairy food isolate could strengthen intestinal barrier function, as demonstrated by an increase in TEER values over the 90 -min incubation period. Previous studies have reported a noticeable increase in TEER values with $L$. plantarum species (Klingberg et al., 2005; Anderson et al., 2010). This is not surprising as different strains of $L$. plantarum can have different effects on membrane integrity studies in vitro. Different L. plantarum strains can either show no increase in TEER value as seen in our study or it can show an increase in TEER value, as evident from studies carried by Klingberg et al. (2005) and Anderson et al. (2010). Most surprising was the observation that the E. coli strain used as a control for this study showed the greatest adherence to Caco-2 cells and either maintained or improved the TEER of the Caco- 2 monolayer exposed to ethanol. This finding suggests that the $E$. coli strain might be a suitable candidate for further evaluation of the characteristics of this bacterium as a probiotic. This information could be used as part of a screening process to identify candidate probiotic bacteria for further investigation into potential health benefits.

In conclusion, our results show that adherence to Caco-2 cells by the bovine rumen isolate L. plantarum $\mathrm{RC} 2$ was not influenced by the presence of E. coli; however, adherence by the dairy food isolate L. rhamnosus MI13 was almost completely inhibited when $E$. coli were present. Conversely, the bovine rumen isolate provided no protection for Caco-2 monolayers exposed to ethanol, whereas the dairy food isolate and E. coli provided either protection or improved integrity of the Caco-2 monolayer exposed to ethanol.

\section{ACKNOWLEDGMENTS}

This work was financially supported by the Faculty of Agriculture and Life Sciences, Lincoln University (Lincoln, New Zealand). The National School of Pharmacy at Otago University (Dunedin, New Zealand) facilitated the work by providing analytical and technical support to complete the Caco-2 cell work.

\section{REFERENCES}

Alander, M., R. Satokari, R. Korpela, M. Saxelin, T. VilpponenSalmela, T. Mattila-Sandholm, and A. von Wright. 1999. Persistence of colonization of human colonic mucosa by a probiotic strain, Lactobacillus rhamnosus GG, after oral consumption. Appl. Environ. Microbiol. 65:351-354.

Anderson, R. C., A. L. Cookson, W. C. McNabb, Z. Park, M. J. McCann, W. J. Kelly, and N. C. Roy. 2010. Lactobacillus plantarum MB452 enhances the function of the intestinal barrier by increasing the expression levels of genes involved in tight junction formation. BMC Microbiol. 10:316.

Bunt, C. R., D. S. Jones, and I. G. Tucker. 1993. The effects of pH, ionic strength and organic phase on the bacterial adhesion to hydrocarbons (BATH) test. Int. J. Pharm. 99:93-98.

Chauvière, G., M. H. Coconnier, S. Kerneis, J. Fourniat, and A. L. Servin. 1992. Adhesion of Lactobacillus acidophilus strain LB to human enterocyte- like Caco2 cells. J. Gen. Microbiol. 138:16891696.

Conway, P. L., S. L. Gorbach, and B. R. Goldin. 1987. Survival of lactic acid bacteria in the human stomach and adhesion to intestinal cells. J. Dairy Sci. 70:1-12.

Darilmaz, D. O., B. Asham, Z. Suludere, and G. Akca. 2011. Influence of gastrointestinal system conditions on adhesion of exopolysaccharide-producing Lactobacillis delbrueckii ssp. bulgaricus strains to Caco-2 cells. Braz. Arch. Biol. Technol. 54:917-926.

Duary, R. K., Y. S. Rajput, V. K. Batish, and S. Grover. 2011. Assessing the adhesion of putative indigenous probiotic lactobacilli to human colonic epithelial cells. Indian J. Med. Res. 134:664-671.

Gao, J. N., E. H. Hugger, M. S. Beck-Westermeyer, and R. T. Borchardt. 2000. Estimating intestinal mucosal permeation of compounds using Caco-2 cell monolayers. Curr. Prot. Pharmacol. $8: 7.2 .1-7.2 .23$

Gueimonde, M., and S. Salminen. 2006. New methods for selecting and evaluating probiotics. Dig. Liver Dis. 38:S242-S247.

Gupta, V., and R. Garg. 2009. Probiotics. Indian J. Med. Microbiol. $27: 202-209$. 
Jose, N. M., C. R. Bunt, and M. A. Hussain. 2015. Comparison of microbiological and probiotic characteristics of lactobacilli isolates from dairy food products and animal rumen contents. Microorganisms $3: 198-212$.

Kleeman, E. G., and T. R. Klaenhammer. 1982. Adherence of Lactobacillus species to human fetal intestinal cells. J. Dairy Sci. 65:2063-2069.

Klingberg, T. D., M. H. Pedersen, A. Cencic, and B. B. Budde. 2005. Application of measurements of transepithelial electrical resistance of intestinal epithelial cell monolayers to evaluate probiotic activity. Appl. Environ. Microbiol. 71:7528-7530.

Liu, X., W. Liu, Q. Zhang, F. Tian, G. Wang, H. Zhang, and W. Chen. 2003. Screening of lactobacilli with antagonistic activity against enteroinvasive Escherichia coli. Food Contr. 30:563-568.

Mattila-Sandholm, T., J. Matto, and M. Saarela. 2009. Lactic acid bacteria with health claims: Interactions and interference with gastrointestinal flora. Int. Dairy J. 9:25-35.
Moussavi, M., and M. C. Adams. 2010. An in vitro study on bacterial growth interactions and intestinal epithelial cell adhesion characteristics of probiotic combinations. Curr. Microbiol. 60:327-335.

Schneeberger, E. E., and R. D. Lynch. 2004. The tight junction: A multifunctional complex. Am. J. Physiol. Cell Physiol. 286:C1213C1228.

Thirabunyanon, M., P. Boonprasom, and P. Niamsup. 2009. Probiotic potential of lactic acid bacteria isolated from fermented dairy milks on antiproliferation of colon cancer cells. Biotechnol. Lett. 31:571-576.

Wang, B., H. Wei, J. Yuan, Q. Li, Y. Li, N. Li, and J. Li. 2008. Identification of a surface protein from Lactobacillus reuteri JCM1081 that adheres to porcine gastric mucin and human enterocyte-like HT-29 cells. Curr. Microbiol. 57:33-38. 